"This is the peer reviewed version of the following article: [BJOG, 2018]. which has been published in final form at [https://obgyn.onlinelibrary.wiley.com/doi/abs/10.1111/14710528.15335]. This article may be used for non-commercial purposes in accordance with Wiley Terms and Conditions for Self-Archiving." 
3

4

\section{Core outcome sets for prevention and treatment of postpartum haemorrhage: an international Delphi consensus study}

Shireen Meher ${ }^{1,2}$, Anna Cuthbert ${ }^{1}$, Jamie J Kirkham ${ }^{3}$, Paula Williamson ${ }^{3}$, Edgardo Abalos ${ }^{4}$, Nasreen Aflaifel $^{1}$, Zulfiqar A Bhutta ${ }^{5}$, Alina Bishop ${ }^{6}$, Jennifer Blum ${ }^{7}$, Peter Collins ${ }^{8}$, Declan Devane ${ }^{9}$, AnneSophie Ducloy-Bouthors ${ }^{10}$, Bukola Fawole ${ }^{11}$, A Metin Gülmezoglu ${ }^{12}$, Kathryn Gutteridge ${ }^{2}$, Gill Gyte ${ }^{6}$, Caroline SE Homer ${ }^{13}$, Shuba Mallaiah ${ }^{14}$, Jeffrey M Smith ${ }^{15}$, Andrew D Weeks ${ }^{1}$, Zarko Alfirevic ${ }^{1}$.

1 Department of Women's and Children's Health, University of Liverpool, UK, 2 Sandwell and West Birmingham Hospitals NHS Trust, Birmingham, UK, 3 Department of Biostatistics, University of Liverpool, UK, 4 Centro Rosarino de Estudios Perinatales (CREP), Rosario, Argentina, 5 Division of Women and Child Health, Aga Khan University, Pakistan, 6 Cochrane Pregnancy and Childbirth Group, University of Liverpool, UK, 7 Gynuity Health Projects, New York, USA, 8 Institute of Infection and Immunity, School of Medicine Cardiff University, UK, 9 HRB-Trials Methodology Research Network, School of Nursing and Midwifery, National University of Ireland Galway, Ireland, 10 Centre Hospitalier Regional Universitaire de Lille, France, 11 Department of Obstetrics and Gynaecology, College of Medicine, University of Ibadan, Nigeria, 12 The UNDP/UNFPA/UNICEF/WHO/World Bank Special programme of research, development and research training in human reproduction (HRP),World Health Organization, Geneva, Switzerland, 13 Centre for Midwifery, Child and Family Health, University of Technology Sydney, Australia, 14 Liverpool Women's Hospital, Liverpool, UK, 15 Jhpiego / Johns Hopkins University, USA.

\section{Corresponding author}

Shireen Meher, +44 (0)7734694660, smeher@liv.ac.uk

\section{Short title: PPH Core Outcome Sets}


44

Objective: To develop core outcome sets (COS) for studies evaluating interventions for (1) prevention and (2) treatment of PPH, and recommendations on how to report the COS.

Design: A two-round Delphi survey and face-to-face meeting.

Population: Health care professionals and women's representatives.

Methods: Outcomes were identified from systematic reviews of PPH studies and stakeholder consultation. Participants scored each outcome in the Delphi on a Likert scale between 1 (not important) and 9 (critically important). Results were discussed at the face-to-face meeting to agree the final COS. Consensus at the meeting was defined as $\geq 70 \%$ of participants scoring the outcome as critically important (7-9). Lectures, discussion and voting were used to agree how to report COS outcomes.

Main outcome measures: outcomes from systematic reviews and consultations.

Results: Both Delphi rounds were completed by 152/205 (74\%) participants for prevention and 143/197 (73\%) for treatment. For prevention of PPH, nine core outcomes were selected: blood loss, shock, maternal death, use of additional uterotonics, blood transfusion, transfer for higher level of care, women’s sense of wellbeing, acceptability and satisfaction with the intervention, breastfeeding and adverse effects. For treatment of PPH, 12 core outcomes were selected: blood loss, shock, coagulopathy, hysterectomy, organ dysfunction, maternal death, blood transfusion, use of additional haemostatic intervention, transfer for higher level of care, women's sense of wellbeing, acceptability and satisfaction with the intervention, breastfeeding and adverse effects. Recommendations were developed on how to report these outcomes where possible.

Conclusions: These COS will help standardise outcome reporting in PPH trials.

Funding: British Medical Association (Strutt and Harper Grant 2014).

Keywords: core outcomes, postpartum haemorrhage, PPH, pregnancy, Delphi

Tweetable abstract: Core outcome sets for PPH: 9 core outcomes for PPH prevention and 12 core outcomes for PPH treatment. 
Over 250,000 women die each year from complications of childbirth. ${ }^{1} \mathrm{PPH}$ is the leading cause of maternal mortality worldwide. ${ }^{2}$ It is usually defined as blood loss of $500 \mathrm{ml}$ or more from the genital tract within 24 hours after childbirth.

Interventions for PPH have been evaluated in a large number of studies. However evidence is difficult to interpret and compare across studies due to variations in the outcomes measured by researchers. In a study looking at outcomes reported in PPH trials published between 1997 and 2015, 121 trials for prevention of PPH used 68 different primary outcomes. ${ }^{3}$ The most commonly reported outcome was assessment of blood loss, with more than ten different cut-offs specified at times ranging from 30 minutes to 48 hours. There were little data on short and long-term morbidity or mortality, and few patient reported outcomes.

Reduction in maternal mortality is part of the Sustainable Development Goals set by the United Nations. ${ }^{1}$ One strategy for achieving this is to ensure that the most effective evidence based therapies are used to manage PPH, and global standards follow evidence based guidelines. ${ }^{1}$ Recommendations can only be robust if they are based on good quality evidence, where interventions are compared using indicators that are standardized and are important measures of wellbeing.

The aim of this project was to develop consensus among international stakeholders on a set of core outcomes that should be used in trials and systematic reviews to evaluate (1) preventative interventions and (2) therapeutic interventions for women with PPH. A secondary aim was to provide guidance on how to report these core outcomes.

\section{METHODS}

The project was registered prospectively with the Core Outcome Measures in Effectiveness Trials (COMET) initiative ${ }^{4}$ and funded by the British Medical Association (BMA). The protocol was peerreviewed by the COMET team and funding body. Ethics approval was not required. ${ }^{5}$ The manuscript has been reported in line with the COS-STAR guidelines for COS reporting. ${ }^{6}$ Methods are summarised in Figure S1 and further details are available in Appendix S1.

The Steering Committee included two obstetricians (SM, ZA), a midwife (AC) and two methodologists/statisticians with expertise in development of COS (JK, PW). A Scientific Advisory Group (SAG), set up to provide multidisciplinary expert input and an international perspective, consisted of 16 members from 10 different countries (Appendix S1). Seven stakeholder groups were agreed a priori to be relevant to the project (obstetricians, midwives, anaesthetists, haematologists, neonatologists, health strategists/methodologists and women's representatives). 
Our aim was to involve as many participants as possible, with a minimum of 10 in each stakeholder group to allow numbers to be meaningful taking into account possible attrition in Round $2 .{ }^{8}$ The same participants were asked to take both the prevention and treatment PPH surveys. Participants were identified from published trial reports and Cochrane reviews on PPH. Invitations were also sent through the CoRe Outcomes in Women's and Newborn health (CROWN) Initiative journal editors mailing list. ${ }^{9}$ Women's representatives were invited through the National Childbirth Trust parent support group (UK) and personal contacts. Further invitations to stakeholders were sent out by snowballing with suggestions from the SAG, authors contacted and targeted participants. The SAG also took a modified Delphi survey separately. This was a methodological investigation to assess the impact of group size on selection of outcomes for a COS, and will be the subject of a separate paper.

\section{Identification of outcomes}

To identify outcomes relevant to PPH, two systematic reviews of randomized trials were undertaken one evaluating interventions for prevention of PPH (NA and ZA), the other, treatment of PPH (SM and ZA) (details in Appendix S1). All published outcomes were considered for inclusion in the COS. .

The reviews identified 121 randomised trials with 160 different outcomes for prevention of PPH and 16 RCTs with 95 different outcomes for treatment of PPH. Outcomes were classified under overarching domains (blood loss assessment, mortality and morbidity, use of additional interventions and resources, women's and clinicians' views, adverse outcomes, and neonatal outcomes). Duplicate outcomes were removed, similar outcomes combined and variations in methods of reporting each outcome noted (Tables S1 and S2). Two outcomes - women's and healthcare professionals' views, were added by the Steering Committee. A total of 35 outcomes for prevention of PPH and 31 outcomes for treatment of PPH were entered into Round 1 of the Delphi.

\section{Delphi Survey}

A two-round, anonymised electronic Delphi survey was designed on DelphiManager ${ }^{10}$ to obtain consensus on the importance of each outcome among stakeholders. It was decided a priori that results of the Delphi would be used to inform the face-to-face stakeholder meeting where a final COS would be agreed.

Each outcome was listed in the survey with its plain language summary. Participants were asked to rate the importance of each outcome between 1 and 9 on a Likert scale, with 1-3 being 'not important', 3-6 'important but not critical' and 7-9 being 'critically important' to report in trials, or select unable to comment. This scale is recommended by the Grading of Recommendations Assessment, Development and Evaluation working group. ${ }^{12}$ Participants were invited to suggest additional outcomes for consideration for the COS in Round 1 using free-text responses. 
Potential participants were invited to register for the study via email, and the Delphi survey was emailed to those who registered. The closing date was set 4 weeks after each round and an e-mail reminder was sent on days 14, 21, and 28. Non-responders in Round 1 were not invited to participate in Round 2. Nonresponders in Round 2 were sent additional emails to improve response rate.

In Round 2, participants were able to view anonymised results from the first round, presented as the distribution of scores for each outcome in each stakeholder group separately. This allowed participants to reflect on their choices prior to completing the second round of the survey. Additional relevent outcomes suggested by participants in Round 1 were were added to Round 2 (16 for prevention and 18 for treatment) (Figures S2 and S3).

We defined consensus for the Delphi a priori based on guidance in The COMET Handbook. ${ }^{8}$ For inclusion in the COS, outcomes required at least $70 \%$ of participants in each stakeholder group to score the outcome as critically important and less than $15 \%$ to score the outcome as not important. Outcomes excluded from the COS required at least $70 \%$ of participants in each stakeholder group to score the outcome as not important and less than $15 \%$ to score the outcome as "critical." If outcomes did not meet either criterion they were classified as outcomes with no consensus.

\section{Face-to-face meeting}

The final phase of the project was a face-to-face consensus meeting (Liverpool, United Kingdom 16-17 ${ }^{\text {th }}$ August 2016). Twenty-five people attended the meeting, and each stakeholder group was represented: five obstetricians, three midwives, four women's representatives, five health strategist/methodologists, one anaesthetist, one haematologist, and one neonatologist (Appendix S1). Findings of the survey were presented and participants were given an opportunity to discuss each outcome. Consideration was given as to whether the outcome was relevant in all setting and for all women recruited. Outcomes could be renamed or reconfigured if there was full consensus at the meeting to do so. Participants then scored each outcome between 1 and 9 on the Likert scale, for inclusion or exclusion in the COS with an anonymous voting system using electronic keypads. Consensus at the meeting required a majority of $70 \%$ of participants to score the outcome as critically important (7-9) to include in the COS.

\section{Methodology for how to report outcomes}

Consensus on how to report the COS outcomes was developed on Day 2 of the meeting by an Expert Committee (n=20; Appendix S1). The aim was not to create new definitions but to select a preferred method of reporting the outcome among existing methods in published literature where possible and to make research recommendations where this was not possible.

We used recommended standards on how to report summary results for trial reporting ${ }^{13}$ and considered the specific metric on how to measure the outcome, the method of data aggregation (continuous or categorical) and the time frame in which to measure the outcome.. Variations in outcome reporting were presented and expert presentations delivered. Options were discussed, and scored. Consensus was defined 
a priori as more than $70 \%$ of participants voting for a preferred option of reporting, and majority view was defined as more than $50 \%$ of participants preferring one option from among the top three options, thereby indicating the strength of the recommendation.

\section{RESULTS}

Survey participants came from 36 different countries (Figure S4), and represented all seven stakeholder groups. For Round 1 of the Delphi, 205 participants responded to the prevention of PPH survey and 197 to the treatment survey. Round 2 was completed by 74\% (152/205) and 73\% (143/197) of participants respectively (Table 1). Overall, 77\% of those who took the survey had exposure to PPH either through personal experience or through caring for women who had experienced PPH. Among women’s representatives, $41 \%$ had experienced a $\mathrm{PPH}$.

Assessment of outcomes for the prevention and treatment of PPH COS is shown in Figure 1. Delphi consensus was reached for including five outcomes in the COS for prevention of PPH and ten outcomes in the COS for treatment of PPH. No outcomes fulfilled criteria for exclusion. There was no consensus on the remaining outcomes. These results were discussed at the face-to face meeting and the final COS agreed included nine outcomes for the prevention COS and 12 outcomes for the treatment COS (Tables 2 and 3). At least one outcome was included from each domain for both prevention and treatment COS, and there was significant overlap in outcomes included in the two COS.

\section{PPH Prevention COS}

For the evaluation of interventions for prevention of PPH, the final COS outcomes are presented in Table 2. At the stakeholder meeting, all outcomes included in the Delphi consensus were included in the COS except for hysterectomy. Although stakeholders at the meeting agreed that hysterectomy was an important outcome, it was not felt to be a critically important outcome in the context of trials for prevention of PPH because it is a very rare event. Although rare but critically important outcomes are also important to include in a COS, and such an outcome of maternal mortality has been included in the prevention of PPH COS, there was consensus that the PPH prevention COS would be more informative if it captured other measures of maternal morbidity for which data were more likely to be available from trials rather than having a COS with little data available for multiple outcomes. Four additional outcomes were included in the PPH prevention COS subsequent to stakeholder discussions and voting. Two of these outcomes, ‘use of blood transfusion' and 'use of additional uterotonics' were borderline for inclusion in the Delphi survey (Table 2). The outcomes 'transfer to ITU' and 'transfer to a higher facility' in the Delphi were reconfigured at the stakeholder meeting to 'transfer to a higher level of care' to capture data on an escalation in the level of care required for the woman, which, it was recognized, would depend on the initial setting of the woman. There was also consensus among stakeholders at the meeting that patient reported outcomes, although not included in the Delphi consensus, were important to include in the COS, and this was strongly advocated by the women's representatives. It was felt that these should capture a woman's sense of wellbeing, as well as acceptability and satisfaction with the 
intervention. Among outcomes in the neonatal domain, there was consensus that breastfeeding would be an important outcome as a PPH may impact on a woman's wellness and ability to establish or maintain breastfeeding if she intended to breastfeed, or there could be a potential impact of the intervention on breast milk itself.

\section{PPH Treatment COS}

For the evaluation of interventions for treatment of $\mathrm{PPH}$, the final COS are presented in Table 3. At the stakeholder meeting, nine of the 10 outcomes included in the Delphi were included in the final COS.. The outcomes 'shock' and 'maternal resuscitation due to shock' were both included by the Delphi survey. However, as both outcomes were very similar, consensus at the meeting was to include 'shock' only in preference to 'maternal resuscitation due to shock' as the latter would be more complex to measure or assess. The outcome disseminated intravascular coagulation, (DIC) was renamed as coagulopathy based on recommendations by haematologists because coagulopathy is the more accurate term; DIC does not have a validated definition in PPH and constitutes only a small subset of coagulopathies associated with PPH. Multiple organ failure was renamed as 'any organ dysfunction', in line with the World Health Organization's recommendations on how to capture severe pregnancy complications including organ dysfunction in the WHO near-miss approach for maternal health. ${ }^{14} \mathrm{~A}$ number of outcomes in the Delphi survey aimed at capturing failure of initial treatment, such as use of additional medical or advanced surgical interventions such as balloon insertion or uterine artery embolisation or ligation. However, at the meeting it was recognized that type of escalation of therapy would depend on the trial intervention itself medical or surgical. Therefore, these outcomes were reconfigured into the outcome 'Use of any additional haemostatic intervention' to capture failure of the trial intervention itself, as this would be applicable to all trials, regardless of the intervention they were evaluating. The other outcomes added were a woman's sense of wellbeing and acceptability and satisfaction with the intervention and breastfeeding as specified above in the prevention of PPH COS.

\section{How to report COS outcomes}

The Expert Committee Recommendations on how to report the COS outcomes are presented in Table 4, along with explanations. Most recommendations were agreed by consensus. Those agreed by a majority view included reporting of hysterectomies specifically carried out to stop PPH, to avoid confounding data with hysterectomies carried out prophylactically or for other indications. For time frames for measuring outcomes, there was consensus that in the context of randomised trials outcome data should be collected from the point of randomisation. The time limit up to which outcomes should be measured was left to trialists for most outcomes to accommodate for local protocols and resource availability. However a majority view recommendation was put forward for blood loss to be assessed (measured or estimated) up to cessation of active bleeding, as this is an area where standardization is particularly lacking and time frame selection is likely to impact on data significantly. For hysterectomy, the majority recommendation was to report it at least up to hospital discharge as most hysterectomies are likely to occur by that time in the context of PPH. 
For the patient reported outcomes and breastfeeding, it was felt by the stakeholder groups that further work was needed to develop tools to capture what aspects of these outcomes were most important to women in the context of a PPH.

\section{DISCUSSION}

\section{Main findings}

Consensus on PPH COS was developed among an international panel of stakeholders through a Delphi survey and face-to-face meeting. For the evaluation of interventions for prevention of PPH, nine core outcomes were selected and for treatment of PPH, 12 core outcomes. Expert committee recommendations where developed on how to report each outcome where possible, and a research agenda was set for two outcomes where this was not possible.

\section{Strengths and Limitations}

This project has several strengths. Firstly, the methodology was defined a priori based on guidelines by the COMET Initiative. ${ }^{8}$ The Delphi exercise has the advantage of including views of a larger number of geographically distant participants. Participants in the Delphi were still able to consider the views of other stakeholder groups in Round 2, to reconsider their opinion without being overly influenced by domineering individuals. Results were further refined at the face-to-face meeting which allowed for rich discussions as well as the ability to debate and persuade others. Secondly, stakeholders came from a range of relevant specialties. Importantly, consumer representatives, who are sometimes overlooked in similar projects, ${ }^{25-26}$ were included at all stages of the process. There was representation from both those who had and had not experienced PPH. Our parent representatives impacted the final COS outcomes by influencing other stakeholders at the face-to-face meeting to include patient reported outcomes. Thirdly, there was representation from a wide range of countries (high, middle and low income) in the Delphi and at the face-to-face meeting, so that the COS developed would be applicable across different settings. Fourthly, we have developed COS for both prevention and treatment of PPH to cover the full spectrum of PPH intervention trials. It is not surprising that there is significant overlap in outcomes selected for the two COS. However the PPH treatment COS appropriately includes more outcomes that would capture significant maternal morbidity in the presence of an established PPH. And finally, a COS often tells researchers what outcomes to use, but not how to report them, making it difficult to achieve adequate standardisation; we have developed Expert Committee Recommendations on how to report the outcomes selected for PPH COS to provide better guidance to researchers.

The limitations of this project are that outcomes were obtained largely from systematic reviews and participants in the Delphi exercise; we did not conduct formal interviews with women. Secondly, we asked participants to identify one key stakeholder group to which they belonged. Some participants may have belonged to more than one stakeholder group and this may have influenced how they scored outcomes, but data are not available to explore this further. Thirdly, representation from each stakeholder groups was not equal; this may have impacted on the outcomes selected. Finally, although we have 
developed guidance on how to report COS outcomes, these recommendations are from a small group of experts, and have not been subjected to the same rigorous Delphi process in a large group. However, it is debatable whether a Delphi process is the optimum method for developing consensus on how to report outcomes. More complex discussions may need to be undertaken for consensus on measurement instruments by stakeholders who may be different from those partaking in the 'what to measure' Delphi. Generic methodological guidelines on how to select standardised instrument measures for outcomes have recently been published. ${ }^{27}$ They recommend identifying all possible measurement instruments for an outcome, and selecting one with high quality of evidence for good validity and internal consistency, that is feasible to measure in the target population. These guidelines have not yet been applied prospectively for COS in maternal health. Our systematic reviews identified the different ways in which COS outcomes have been reported, and the feasibility of applying the instruments in an international setting was considered when making recommendations on how to report outcomes. While validity may be excellent for the more objective outcomes included in the COS, such as units of blood transfusion or maternal death, measurement tools for other outcomes such as blood loss are well known for their poor accuracy.

\section{Interpretation}

To our knowledge, there are no other published COS for PPH. These COS include outcomes that capture meaningful morbidity (shock, hysterectomy, organ dysfunction) and mortality. They also include outcomes with high event rates upon which sample size calculations could be based for smaller studies (blood loss, use of additional uterotonics or blood transfusion). Resource use may be assessed through use of additional interventions and level of care such as ITU use. Patient reported outcomes (woman's sense of wellbeing, acceptability/satisfaction with the intervention) and breastfeeding are also captured but require further qualitative research on how best to measure these outcomes. Until further data are available, we would encourage researchers to clearly report the measures they have used.

Although these outcomes aim to assess the impact of interventions evaluated on severity of PPH, some outcomes may be influenced by local practices. For example, thresholds used for transfusing blood or transferring a woman for higher level of care may vary across trial settings and studies. For such outcomes we would encourage authors to interpret results bearing the potential impact of local practices in mind.

We would recommend researchers evaluating interventions for PPH to report these COS outcomes as a minimum, along with any other outcomes of interest to their study. Where these COS are not reported, researchers are encouraged to provide an explanation, for transparency and to reduce the risk of reporting bias. Future trials evaluating interventions for PPH should report any barriers identified to data collection for these COS outcomes. COS may be updated to provide guidance in response to such feedback.

\section{CONCLUSIONS}

The PPH COS, developed through an international multidisciplinary effort, will help standardise outcome reporting in this area, and facilitate comparison of data across studies, to guide clinical practice. We 
recommend that researchers evaluating interventions for PPH prevention and treatment should report these COS outcomes as a minimum, along with any other outcomes of interest. Further work is needed on how to best to report women's sense of wellbeing, acceptability and satisfaction with the intervention and breastfeeding in the context of PPH.

\section{ACKNOWLEDGEMENTS}

We would like to acknowledge (1) participants of the Delphi survey (Appendix S2); (2) participants at the consensus meeting not in the authorship: Jim Neilson (chair), Joao Paulo Souza, Halima Shakur, Beverely Hunt, Julie Nycyk, Steven Lane, Michelle Beacock, Helen Castledine, Carolyn Markham; (3) the National Childbirth Trust, UK for facilitating women's representation; (4) the Cochrane Pregnancy and Childbirth Group for facilitating the development of systematic reviews; (5) the COMET Initiative team for methodological support (6) the CROWN Initiative for circulating the Delphi survey and (7) the World Health Organisation for supporting and endorsing the project.

\section{DISCLOSURE OF INTERESTS}

The instrument for measurement of organ dysfunction and coagulopathy was adopted from the published WHO tool for assessing maternal morbidity. ${ }^{14}$ Although representatives from the WHO were involved in the project, this was a consensus decision by all participants at the meeting as it was felt that the tool was developed through a rigorous process, was feasible to apply in all settings and there were no better established alternative tools.

\section{CONTRIBUTION OF AUTHORSHIP}

SM and ZA conceived the idea and developed the protocol with PW. SM and AC executed the project with input from JK, PW and ZA. EA, NA, ZAB, AB, JB, PC, DD, AD-B, BF, AMG, KG, GG, CSEH, ShuM, JMS, ADW all took the SAG Delphi and provided expert input at various stages of the project. Data were analysed by AC and SM with input from JK and PW. SM wrote the manuscript with input from all co-authors.

\section{DETAILS OF ETHICS APPROVAL}

Ethics approval was not required as assessed by the MRC HRA tool ${ }^{5}$ as the study was not a clinical trial, did not assess a devise or expose patient to ionising radiation, did not require collection or storage of any material / specimens / protected information, recruit patients / carers through the NHS, involve anyone 
with lack of capacity or prisoners, or xenotransplantation, and was not a social care project funded through the Department of Health, UK.

\section{FUNDING}

This project was funded by the British Medical Association Strutt and Harper Grant. The funders reviewed the application and protocol prior to awarding the grant, monitored the project progress, and provided financial support to disseminate the COS through international meetings but were not directly involved in any other aspect of the project.

\section{REFERENCES}

1. Word Health Organization. Fact sheet: Maternal Mortality. Available from: http://www.who.int/mediacentre/factsheets/fs348/en/ [Accessed 16 $6^{\text {th }}$ January 2017].

2. Say L, Chou D, Gemmill A, Tunçalp Ö, Moller AB, Daniels JD, et al. Global Causes of Maternal Death: A WHO Systematic Analysis. Lancet Global Health. 2014;2(6): e323-e333. doi: 10.1016/S2214-109X(14)70227-X.

3. Aflafeil, N. Postpartum haemorrhage: New insights from published trials and the development of new management operations_[PhD dissertation]. Liverpool: University of Liverpool; 2015.

4. COMET Initiative. Core Outcome Sets for Prevention and Treatment of Postpartum Haemorrhage. Registration number 703. Available from: http://www.cometinitiative.org/studies/details/706) [Accessed $1^{\text {st }}$ March 2015].

5. Medical Research Council. Health Research Authority: Do I need NHS REC approval. Available from: http://hra-decisiontools.org.uk/ethics/ [Accessed $1^{\text {st }}$ March 2014].

6. Kirkham JJ, Gorst S, Altman DG, Blazeby JM, Clarke M, Devane D, et al. Core Outcome SetSTAndards for Reporting: The COS-STAR Statement. PLoSMed 2016;13(10):e1002148.doi:10.1371/journal.pmed.100214.

7. The World Bank. Country classification: World Bank Country and Lending Groups. Available from: https://datahelpdesk.worldbank.org/knowledgebase/articles/906519 [Accessed 1st June 2017].

8. Williamson, PR, Altman DG, Bagley H, Barnes KL, Blazeby JM, Brookes, ST, et al. The COMET Handbook: Version 1.0. Available from: https://trialsjournal.biomedcentral.com/track/pdf/10.1186/s13063-017-19784?site=trialsjournal.biomedcentral.com [Accessed 15 ${ }^{\text {th }}$ July 2017].

9. CROWN: Core Outcomes in Women's and Newborn Health. Available from: http://www.crowninitiative.org/ [Accessed 15 $5^{\text {th }}$ June 2017].COMET Initiative.

10. Delphi Manager. Available from: http://www.comet-initiative.org/delphimanager/ [Accessed May 2017]. 
11. Readability Formulas. The SMOG Readability Formula, a Simple Measure of Gobbledygook. Available from: http://www.readabilityformulas.com/smog-readability-formula.php [Accessed 1st November 2015].

12. Guyatt GH, Oxman AD, Kunz R, Atkins D, Brozek J, Vist G, et al. GRADE guidelines: 2. Framing the question and deciding on important outcomes. J Clin Epidemiol 2011;64:395-400.

13. Zarin DA, Tse T, Williams RJ, Califf RM, Ide NC. The ClinicalTrials.gov results database-update and key issues. N Engl J Med. 2011;364(9):852-60.

14. Evaluating the quality of care for severe pregnancy complications: the WHO near-miss approach for maternal health. World Health Organisation 2011. Available from: http://apps.who.int/iris/bitstream/10665/44692/1/9789241502221 eng.pdf [Accessed 15th June 2016].

15. Schorn MN. Measurement of blood loss: review of the literature. J Midwifery Womens Health. 2010;55(1):20-7. doi: 10.1016/j.jmwh.2009.02.014.

16. Ambardekar S, Shochet T, Bracken H, Coyaji K, Winikoff B. Calibrated delivery drape versus indirect gravimetric technique for the measurement of blood loss after delivery: a randomized trial. BMC Pregnancy Childbirth. 2014;14:276. doi: 10.1186/1471-2393-14-276.

17. Zhang W, Deneux-Tharaux C, Brocklehurst P Juszczak E, Joslin M, Alexander S on behalf of the EUPHRATES Group. Effect of a collector bag for measurement of postpartum blood loss after vaginal delivery: cluster randomised trial in 13 European countries BMJ 2010;340:c293 doi:10.1136/bmj.c293.

18. Diaz V, Abalos E, Carroli G. Methods for blood loss estimation after vaginal birth. Cochrane Database of Systematic Reviews 2014, Issue 2. Art. No.: CD010980. DOI: 10.1002/14651858.CD010980.

19. Kohn JR, Dildy GA, Eppes CS. Shock index and delta-shock index are superior to existing maternal early warning criteria to identify postpartum hemorrhage and need for intervention. J Matern Fetal Neonatal Med. 2017 Nov 7:1-241. doi: 10.1080/14767058.2017.1402882.

20. Nathan HL, Cottam K, Hezelgrave NL, Seed PT, Briley A, Bewley S, et al. If Determination of Normal Ranges of Shock Index and Other Haemodynamic Variables in the Immediate Postpartum Period: A Cohort Study. PLoS One. 2016 Dec 20;11(12):e0168535. doi: 10.1371/journal.pone.0168535. eCollection 2016.

21. Nathan HL1, El Ayadi A, Hezelgrave NL, Seed P, Butrick E, Miller S, et al. Shock index: an effective predictor of outcome in postpartum haemorrhage? BJOG. 2015 Jan;122(2):268-75. doi: 10.1111/14710528.13206 .

22. El Ayadi AM, Nathan HL, Seed PT, Butrick EA, Hezelgrave NL, Shennan AH, et al. Vital Sign Prediction of Adverse Maternal Outcomes in Women with Hypovolemic Shock: The Role of Shock Index.. PLoS One. 2016 Feb 22;11(2):e0148729. doi: 10.1371/journal.pone.0148729. eCollection 2016.

23. Pacagnella R, Souza JP, Durocher J, Perel P, Blum J, Winikoff B, et al A Systematic Review of the Relationship between Blood Loss and Clinical Signs. 2013. PLoS ONE 8(3): e57594. doi:10.1371/journal.pone.0057594.

24. CRADLE-2. CRADLE Trial. Available from http://cradletrial.com/2-wordwide-study/ [Accessed $22^{\text {nd }}$ February 2018]. 
25. Gargon E, Gurung B, Medley N, Altman DG, Blazeby JM, Clarke M, et al. Choosing important health outcomes for comparative effectiveness research: a systematic review. PLoS One 2014;9(6):e99111. doi: 10.1371/journal.pone.0099111. eCollection 2014.

26. Gorst SL, Gargon E, Clarke M, Blazeby JM, Altman DG, Williamson PR. Choosing Important Health Outcomes for Comparative Effectiveness Research: An Updated Review and User Survey. PLoS One. 2016;11(1):e0146444. doi: 10.1371/journal.pone.0146444. eCollection 2016. measurement instruments for outcomes included in a "Core Outcome Set" - a practical guideline. 